\title{
Barreiras à implementação de recomendações ao parto normal no Brasill: a perspectiva das mulheres
}

\author{
Ávila Teixeira Vidal, ${ }^{1}$ Jorge Otávio Maia Barreto² e Daphne Rattner ${ }^{1}$
}

Como citar Vidal AT, Barreto JOM, Rattner D. Barreiras à implementação de recomendações ao parto normal no Brasil: a perspectiva das mulheres. Rev Panam Salud Publica. 2020;44:e164. https://doi.org/10.26633/RPSP.2020.164

RESUMO

Objetivo. Identificar barreiras à implementação das diretrizes de assistência ao parto normal no Brasil sob a perspectiva das mulheres.

Métodos. Realizou-se um estudo descritivo-exploratório, de abordagem qualitativa e perspectiva interpretativista. A unidade hermenêutica foi construída a partir das contribuições de usuárias a uma consulta pública on-line sobre as Diretrizes Nacionais de Assistência ao Parto Normal realizada em 2016 pela Comissão Nacional de Incorporação de Tecnologias no Sistema Único de Saúde (CONITEC). Foi utilizada a metodologia de análise de conteúdo para examinar especificamente as respostas à questão "Considerando sua realidade local, o que dificultaria a implantação deste protocolo ou diretriz?".

Resultados. Das 396 contribuições recebidas pela CONITEC, 55 foram incluídas na análise de conteúdo. A média de idade das mulheres foi de 31 anos, sendo a maioria branca (69\%) e residente na região Sudeste do Brasil (56,3\%). A codificação revelou sete categorias de barreiras, agrupadas em três famílias — barreiras relacionadas a 1) formação e cultura profissional (com destaque para a centralidade dos médicos, e não das mulheres, no parto), 2) cultura social (falta de informação por parte da população) e 3) questões políticas e de gestão (falta de interesse dos gestores, menor remuneração para médicos que atendem parto normal vs. cesariana e falta de infraestrutura hospitalar).

Conclusões. Os resultados mostraram que aspectos da formação e cultura profissional, cultura social e questões políticas e de gestão são pontos críticos que devem ser considerados na realização de intervenções futuras com o objetivo de transpor ou enfraquecer as barreiras à implementação de recomendações ao parto normal no Brasil.

Palavras-chave Parto; protocolos clínicos; ciência da implementação; Brasil.

O Brasil possui um dos maiores e mais complexos sistemas de saúde pública de acesso universal do mundo, o Sistema Único de Saúde (SUS), que garante assistência integral desde a atenção primária até a alta complexidade, incluindo programas de imunização, acesso a medicamentos de alto custo e transplante de órgãos (1). De forma suplementar, pouco mais de 46 milhões dos 209,5 milhões de brasileiros possuem planos privados de saúde (2).

Nas últimas décadas, o Brasil, assim como outros países latino-americanos, vivenciou um crescimento alarmante das taxas de cesariana, tornando-se essa a principal via dos nascimentos ocorridos - 55,9\% em 2018 (3). Nesse mesmo ano, a taxa média de cesariana chegou a $70 \%$ em algumas unidades da federação e a $90 \%$ em algumas unidades de saúde privadas $(3,4)$.

Para enfrentar essa problemática e qualificar a atenção ao parto e nascimento, várias ações têm sido articuladas na esfera tanto do sistema público quanto do sistema de saúde suplementar $(5,6)$, com destaque para as Diretrizes Nacionais de Assistência ao Parto (7). Essas Diretrizes, elaboradas pelo Ministério da

\footnotetext{
1 Universidade de Brasília (UnB), Faculdade de Ciências da Saúde, Departamento de Saúde Coletiva, Brasília (DF), Brasil.
} 
Saúde do Brasil em parceria com sociedades científicas e representantes da sociedade civil, propõem recomendações baseadas em evidências sobre questões relacionadas às vias de parto. A elaboração das Diretrizes seguiu métodos sistemáticos descritos na literatura para adaptação à realidade local de diretrizes clínicas já existentes (8). Tal processo foi conduzido pela Comissão Nacional de Incorporação de Tecnologias no Sistema Único de Saúde (CONITEC), responsável legal (9) por assessorar a elaboração de Protocolos Clínicos e Diretrizes Terapêuticas do Ministério da Saúde.

Apesar desses esforços metodológicos, as Diretrizes possuem fragilidades. Na aplicação do instrumento Appraisal of Guidelines for Research E Evaluation II (AGREE II) (10), utilizado na avaliação do rigor metodológico de diretrizes clínicas, observou-se que o domínio da "aplicabilidade", que diz respeito à análise e explicitação dos potenciais fatores para implementação das diretrizes, foi pouco atendido.

A experiência internacional aponta que, além do planejamento da implementação já no momento da formulação de diretrizes clínicas $(11,12)$, a inclusão da perspectiva dos usuários em todas as etapas da elaboração também é fundamental, possibilitando a incorporação de experiências e demandas. Além disso, a inclusão da perspectiva de usuários tem um papel importante na disseminação do conhecimento relacionado com o tema abordado $(13,14)$.

Para inclusão da perspectiva da sociedade em seu processo de decisão, a CONITEC tem estimulado a participação social por meio de consultas públicas, com a disponibilização de formulário específico para contribuições de usuários (15). Nesse contexto, o objetivo do presente estudo foi identificar potenciais barreiras à implementação das Diretrizes Nacionais de Assistência ao Parto Normal no Brasil a partir da perspectiva das mulheres. Tal perspectiva se justifica por serem as mulheres as personagens principais e diretamente afetadas por essas recomendações.

\section{MATERIAIS E MÉTODOS}

Este estudo integrou o projeto "Barreiras e estratégias para a implementação das Diretrizes do Parto Normal no Brasil", no âmbito da iniciativa Embedding Research for the Sustainable Development Goals (ER-SDG), da Organização Pan-Americana da Saúde (OPAS) e instituições parceiras. Outros dois artigos relacionados também compõem este suplemento especial $(16,17)$.

Foi realizado um estudo descritivo-exploratório, de abordagem qualitativa e perspectiva interpretativista, cujo objeto de análise foi a contribuição das mulheres participantes da Consulta Pública 01/2016, que tratou da versão preliminar das Diretrizes Nacionais de Assistência ao Parto Normal (7). O documento foi disponibilizado no portal público da CONITEC e recebeu contribuições por formulário on-line de 12 de janeiro a 29 de fevereiro de 2016. Após esse período, todas as contribuições recebidas foram disponibilizadas integralmente, de forma anônima, no portal eletrônico da CONITEC (18). Para o presente estudo, foram excluídas as contribuições de profissionais de saúde, pessoas interessadas no tema e pessoas jurídicas, sendo incluídas apenas as respostas de usuárias (18) do sexo feminino à seguinte pergunta: "Considerando sua realidade local, o que dificultaria a implantação deste protocolo ou diretriz?".

Após a organização dos dados em uma planilha eletrônica, foi conduzida análise de conteúdo das contribuições. A análise foi realizada em três etapas, de acordo com os fundamentos propostos por Bardin (19). Primeiro, foi realizada a pré-análise do conteúdo, momento em que foram organizadas as contribuições por tipo e selecionadas aquelas advindas de mulheres, a fim de criar uma unidade hermenêutica. Na segunda etapa, de exploração do material, deu-se a leitura e a codificação dos dados. A terceira etapa enfocou o tratamento dos resultados e sua interpretação, para categorização do conteúdo conforme características comuns. Essa categorização foi realizada de forma não apriorística, ou seja, as categorias emergiram da interpretação dos sentidos obtidos nas codificações. Posteriormente, as categorias foram organizadas em grupos, denominados de "famílias", de acordo com as relações percebidas entre elas. A análise de conteúdo foi conduzida com apoio do software Atlas.Ti (7.0).

Foram adotados critérios para avaliação do rigor metodológico em pesquisa qualitativa (credibilidade, confiabilidade, confirmabilidade e transferibilidade) (20). A fim de garantir a credibilidade e a confiabilidade dos achados, os dados utilizados neste estudo estão disponíveis em um repositório público de dados (http://dx.doi.org/10.17632/znjsyz3kzy.1). Para garantir a validade, a análise dos dados foi realizada por uma pesquisadora (ATV) e validada a posteriori por outra pesquisadora (DR). O critério de confirmabilidade foi atendido pelo uso do software Atlas.ti, uma vez que o registro de todas as etapas metodológicas realizadas na pesquisa está disponível em um repositório público de dados, no qual é possível acessar o relatório com as codificações das citações e a classificação das famílias identificadas na unidade hermenêutica (http:// dx.doi.org/10.17632/znjsyz3kzy.1). Com relação à transferibilidade, ressalta-se que as contribuições das mulheres podem sofrer efeitos do contexto, ou seja, o fato de terem sido obtidas a partir de uma consulta pública, anônima, via Internet, em um dado período de tempo, pode afetar o conteúdo das contribuições.

Compreende-se que um processo de reflexividade é importante para o reconhecimento da influência dos valores dos pesquisadores na interpretação dos dados. Apesar de não ter ocorrido nenhum momento de interação entre o grupo de pesquisa e as mulheres que realizaram as contribuições, admite-se que o lugar de onde se observa o objeto de estudo pode afetar a interpretação dos dados. É importante, assim, declarar que as pesquisadoras responsáveis pela análise dos dados participaram do processo de discussão das recomendações das Diretrizes e que uma delas atua na defesa de temas relacionados com a saúde da mulher e a humanização do parto. Assim, embora a experiência e o conhecimento das temáticas abordadas tenham auxiliado a identificação e o reconhecimento das categorias, expressam também uma perspectiva de análise.

De acordo com a Resolução $n^{\circ}$ 510/2016 do Conselho Nacional de Saúde (CNS), considerando que o conteúdo analisado é de caráter público e sem identificação (18), foi dispensada a aprovação de Comitê de Ética em Pesquisa.

\section{RESULTADOS}

Foram recebidas 396 contribuições pela CONITEC, das quais 66 eram procedentes de usuários/pacientes, 24 de familiares, amigos ou cuidadores de paciente, 233 de profissionais de saúde, 63 de interessados no tema e 10 de pessoas jurídicas. Das 90 contribuições procedentes do formulário de usuários/pacientes, amigos ou cuidadores, 82 eram de mulheres. Dessas, 55 responderam à pergunta do questionário "Considerando sua realidade local, o 
FIGURA 1. Rede de relações entre as famílias de dificuldades apontadas por mulheres participantes de uma consulta pública sobre implementação das recomendações ao parto normal no Brasila

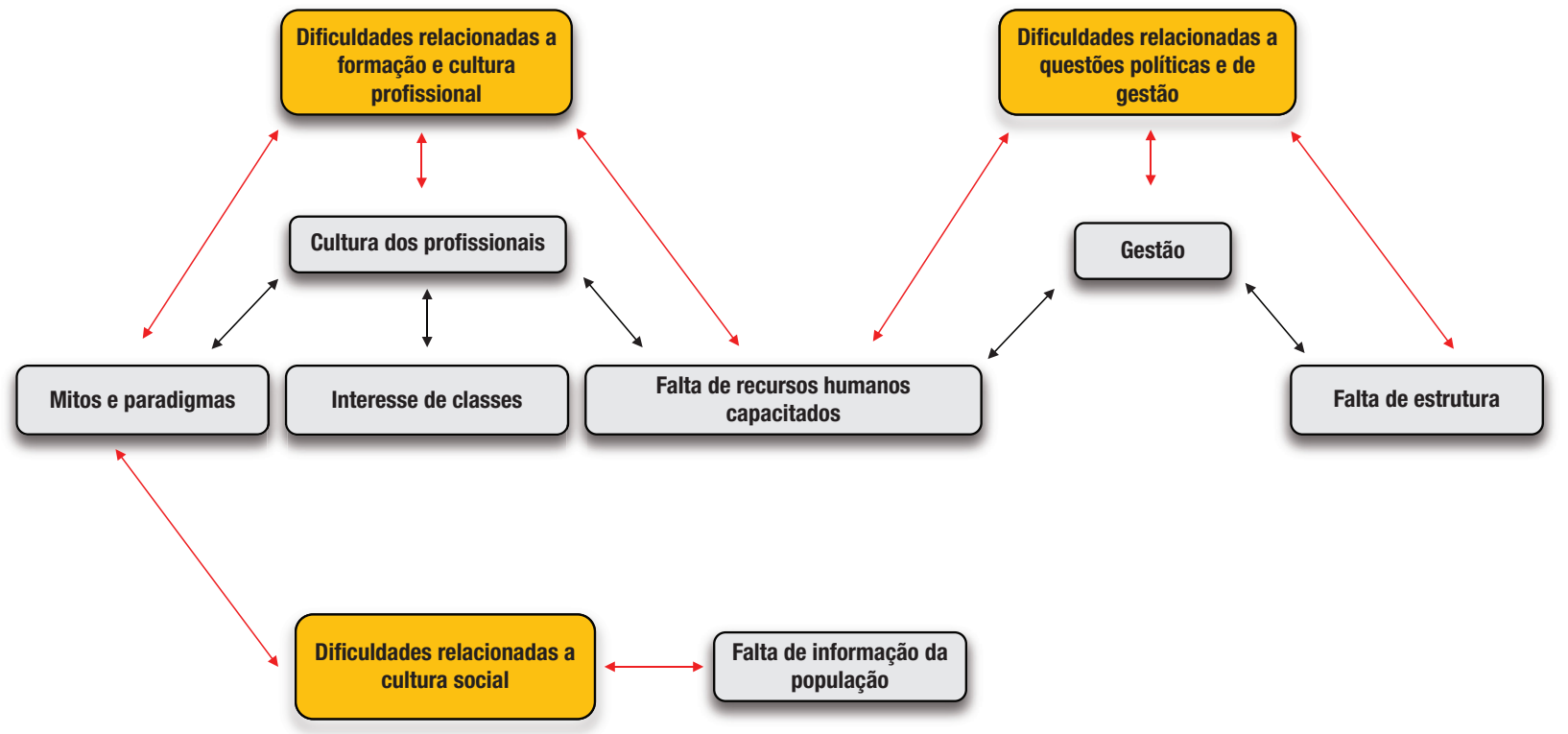

${ }^{a}$ As famílias de dificuldades aparecem em laranja. Setas vermelhas representam a relação entre as famílias de dificuldades e as codificações; setas pretas representam a relação entre as codificações.

que dificultaria a implantação deste protocolo ou diretriz?". Essas respostas foram submetidas à análise de conteúdo.

A média de idade dessas mulheres era de 31 anos, sendo a maioria branca $(69 \%)$ e residente na região Sudeste do Brasil (56,3\%). Ao analisar o conteúdo das contribuições das mulheres sobre as dificuldades de implementação das diretrizes para o parto normal, foram identificadas sete categorias recorrentes nos seus discursos: 1) cultura dos profissionais; 2) falta de recursos humanos capacitados; 3) gestão; 4) falta de estrutura; 5) falta de informação da população; 6) interesse de classes; e 7) mitos e paradigmas.

Essas categorias foram agrupadas em três famílias, relacionadas a 1) formação e cultura profissional, 2) cultura social e 3) questões políticas e de gestão. Observaram-se inter-relações entre as famílias apresentadas, sendo as categorias que estabelecem esses elos a falta de recursos humanos capacitados e os mitos e paradigmas, como mostra a figura 1.

\section{Dificuldades relacionadas a formação e cultura profissional}

Sobre a cultura dos profissionais, destacaram-se, nas contribuições das mulheres, a resistência dos profissionais de saúde de aceitar mudanças, a centralidade dos médicos, e não das mulheres, no parto, e a falta de autonomia de outros profissionais não médicos. Esses aspectos são exemplificados nos depoimentos a seguir:

A resistência dos/as profissionais à mudança da realidade. Enquanto a postura dos/as profissionais ainda for no sentido de inviabilizar a humanização do parto, seja por conveniência, falta de paciência para a espera do desencadeamento e andamento normal do trabalho de parto, a implementação do protocolo ou diretriz será cada vez mais distante de sua concretização. (CONITEC, Consulta Pública 01/2016, p. 126) (18)
Os profissionais da área da saúde (obstetras, técnicos em enfermagem, enfermeiros e pediatras) insistem em tornar o parto um evento médico [...]. Não deixam a mulher ser protagonista, caminhar, se alimentar ou escolher a posição mais confortável para parir. (CONITEC, Consulta Pública 01/2016, p. 15) (18)

Dois outros pontos se destacam nessa categoria - a questão da humanização e a violência obstétrica — ambos abordados com ênfase na falta de tratamento humanizado, com intervenções violentas que desrespeitam os direitos da mulher:

Muitas violências obstétricas ainda acontecem no contexto obstétrico atual, que nos deixam indignadas, e sabemos, com poucas informações que temos como leigas, que não precisam ser assim os atendimentos às gestantes. (CONITEC, Consulta Pública 01/2016, p. 111) (18)

Outras três categorias que se relacionam com a questão da cultura dos profissionais são a falta de recursos humanos capacitados, interesses de classes e mitos e paradigmas. O conteúdo das contribuições das mulheres segue no sentido de afirmar que faltam, na rede, profissionais de saúde capacitados para um atendimento integral e humanizado, com um olhar para as práticas baseadas em evidências científicas e não baseadas em mitos e paradigmas assistenciais ultrapassados. Isso é reforçado pelos interesses de classes, que, segundo a contribuição das mulheres, perpetuam essas práticas e esse olhar sobre o campo do parto e nascimento ao defender certas práticas e modelos de assistência ao parto:

[...] um grande dificultador serão as corporações médicas, que desejam manter o controle do corpo da mulher e os procedimentos que vêm adotando há décadas, privilegiando a cesárea, a episiotomia e a não informação esclarecida da mulher assistida 
na hora do parto. (CONITEC, Consulta Pública 01/2016, p. 227) (18)

Cabe aqui a reflexão sobre a normatização da concessão do "pedido" da realização de cesariana pelas mulheres, o real desejo dessas mulheres e a necessidade de se realizar essa intervenção. Essa situação pode exemplificar o que as mulheres expressaram, em sua contribuição à consulta pública, como interesses de classes.

\section{Dificuldades relacionadas a cultura social}

Um ponto importante levantado como fragilidade para o exercício pleno da autonomia e reinvindicação de direitos foi a falta de informações da população, que apareceu em contribuições como "desconhecimento e falta de informação da população", e preocupação quanto à "acessibilidade desta proposta às gestantes e aos pais".

Para fins desta análise, consideraram-se os mitos e paradigmas como aspectos que reforçam essa questão, como indicam as menções a "antigos conceitos e preconceitos" e "paradigmas e mitos culturais pregados há décadas".

\section{Dificuldades relacionadas a questões políticas e de gestão}

Muito presentes também nas contribuições dessas mulheres foram questões relacionadas à gestão como fator para dificultar a implementação de uma política de apoio à assistência ao parto normal de qualidade, tendo em vista, segundo as contribuições, a "falta de vontade política dos gestores" (p. 30), "falta de interesse da prefeitura e da secretaria de saúde" (p. 34) e "... manobras políticas [que] impedem que muitos recursos sejam implantados em meu município" (p. 136) (CONITEC, Consulta Pública 01/2016 (18).

Outra questão que emergiu na contribuição das mulheres sobre a gestão foi a forma de remuneração dos médicos:

A remuneração dos profissionais que assistem o parto normal, pois é muito mais vantajoso fazer 10 cesáreas no dia do que atender somente um trabalho de parto que dure 12 horas. (CONITEC, Consulta Pública 01/2016, p. 23) (18)

O pagamento realizado aos médicos pelos planos de saúde deve ser justo, ou seja, um valor muito acima do empregado nos dias atuais. (CONITEC, Consulta Pública 01/2016, p. 246) (18)

Além disso, outro destaque nas contribuições é a falta de fiscalização e cumprimento da Resolução da Diretoria Colegiada (RDC) 36 da Agência Nacional de Vigilância Sanitária (ANVISA), que regulamenta o funcionamento dos serviços de atenção obstétrica e neonatal, e da Lei do Acompanhante (21), que garante às parturientes o direito à presença de um acompanhante durante o trabalho de parto, parto e pós-parto imediato: “... a falta de fiscalização do cumprimento da RDC 36/2008 e da Lei do Acompanhante" (p. 181).

A falta de estrutura foi sinalizada como falta de profissionais, instalações hospitalares inadequadas ou mesmo ausência de maternidades. Outro problema de estrutura relatado foi a ausência de casas de parto e escassez de enfermeiras obstétricas e obstetrizes: "A falta de casas de parto e de acesso a enfermeiras obstétricas e obstetrizes que possam acompanhar as gestantes" (CONITEC, Consulta Pública 01/2016, p. 235).

\section{DISCUSSÃO}

O presente estudo analisou a contribuição das brasileiras participantes de um processo de consulta pública acerca das Diretrizes Nacionais de Assistência ao Parto Normal, com foco nas dificuldades percebidas por essas usuárias para a implementação das Diretrizes. Entre as dificuldades citadas, os depoimentos acerca de dificuldades relacionadas a formação e cultura profissional vão ao encontro de relatos anteriores. $\mathrm{O}$ inquérito nacional "Nascer no Brasil", que entrevistou 23894 mulheres de todas as regiões do país sobre intervenções obstétricas durante o trabalho de parto e parto (22), observou boas práticas durante o trabalho de parto em menos de $50 \%$ das experiências das mulheres. Em estudo realizado em 2017, com todas as 606 maternidades públicas e mistas envolvidas na Rede Cegonha e um total de 10675 puérperas, Leal et al. (23) observaram frequências menores às da pesquisa Nascer no Brasil, mas ainda expressivas, de práticas consideradas prejudiciais, como episiotomia ( $27,7 \%$ vs. $47,3 \%)$ e manobra de Kristeller (15,9\% vs. $36,1 \%)$.

Para evidenciar o reconhecimento internacional do problema de práticas prejudiciais no momento do parto, a Organização Mundial da Saúde (OMS) publicou, em 2015, declaração sobre a prevenção e eliminação do desrespeito e abuso durante o parto (24). Além disso, esse tema aparece como recomendação explícita tanto em diretrizes da OMS sobre cuidados intraparto para uma experiência positiva de parto (25) como na International Childbirth Initiative (ICI) (26), uma parceria com a Federação Internacional de Ginecologia e Obstetrícia (FIGO). Essa discussão é tão relevante e atual que, em 2019, uma revisão sistemática com estudos da América Latina relatou prevalência de $43 \%$ de desrespeito e abuso às mulheres durante o parto, evidenciando que essa violência é da cultura dos serviços (27).

No Brasil, diante de um cenário de discussão sobre a autonomia das mulheres e do profissional médico, o Conselho Federal de Medicina (CFM) resolveu se manifestar por meio de uma Resolução (28) que diz, no seu artigo $1^{\circ}$, que, desde que tenha recebido as informações sobre os riscos e benefícios do parto vaginal e cesariana, é direito da gestante optar pela realização de cesariana em situações eletivas (a partir da $39^{\mathrm{a}}$ semana).

Dados da pesquisa Nascer no Brasil (22) revelaram que a preferência das mulheres pela cesariana no início da gestação foi de apenas 27,6\%, com variações a depender do setor (público ou suplementar) em que estavam sendo assistidas e do número de gestações anteriores. É importante destacar que, independentemente do desejo das mulheres e do setor de assistência, a proporção de cesáreas foi superior à preferência inicial dessas mulheres.

Em estudo realizado em maternidades do Sul do Brasil (29), observou-se que os fatores significativamente associados ao pedido de realização da cesariana foram: elevada escolaridade e renda familiar, realização de pré-natal no setor privado, ter sido atendida pelo mesmo médico durante todo o pré-natal e não ter referido comorbidade no período gestacional. Ou seja, a ocorrência de cesarianas a pedido foi maior em mulheres com menores riscos de complicações durante a gravidez e o parto.

Quanto às dificuldades relacionadas à cultura social, a falta de informação e os mitos e paradigmas apareceram nos depoimentos. Em consonância, as recomendações da OMS sobre 
Intervenções Não Clínicas para Reduzir Cesarianas Desnecessárias (30) destacam que a educação em saúde para as mulheres é um componente essencial no cuidado pré-natal, pois elas se sentem fortalecidas, o que fornece, inclusive, a base para um diálogo informado com os profissionais de saúde.

Um exemplo de como a falta de informação pode afetar a implementação das Diretrizes pode ser observado no documento da OMS sobre cuidados intraparto (25). Esse documento relata que algumas mulheres podem apresentar dificuldades em relação ao uso de analgesia epidural por medo do procedimento e de seus riscos, dor, conflito ou culpa. Ao compreender que o fenômeno de altas taxas de cesariana no Brasil e outros países da América Latina tem forte relação com fatores socioculturais (31), ressalta-se aqui a importância da comunicação eficaz e do acesso à informação para essas mulheres.

Em relação a dificuldades relacionadas a questões políticas e de gestão, cabe lembrar que o apoio contínuo à mulher durante o trabalho de parto, parto e pós-parto imediato, por um acompanhante, é direito garantido por lei no Brasil desde 2005 (21); no entanto, observa-se que, passados 15 anos desse marco legal, ainda há dificuldades com relação ao seu cumprimento. Diniz et al. (32) observaram que $24,5 \%$ das mulheres não tiveram acompanhamento em nenhum momento da internação hospitalar, com diferenças importantes no setor público $(29,5 \%)$ e privado $(4,7 \%)$.

O estudo de Bittencourt et al. (33) evidenciou que, no Brasil, $70,6 \%$ e $98,8 \%$ dos hospitais públicos e privados, respectivamente, dispunham de equipamentos mínimos de emergência materna. Com relação aos equipamentos mínimos de emergência para recém-nascidos, a proporção foi de $67,7 \%$ e $87,8 \%$, respectivamente. Essas diferenças aumentavam regionalmente. Os hospitais públicos das regiões Norte e Nordeste apresentavam percentuais de $56,3 \%$ e $44,8 \%$ de disponibilidade de equipamentos de emergência materna e de recém-nascidos. Essa situação explicita o problema de inadequação da estrutura hospitalar, que é barreira importante à implementação de diretrizes assistenciais que objetivam a qualidade do cuidado. No estudo de Bittencourt et al., foi utilizada, como indicador para avaliação dos recursos humanos, a verificação da existência de coordenador médico e de enfermagem com título de especialista nos serviços de obstetrícia e neonatologia; observou-se que, entre médicos coordenadores, a maioria possuía especialização em obstetrícia — 94,4\% nos hospitais públicos e 100,0\% nos hospitais privados. Já na neonatologia, os percentuais foram $94,4 \%$ e 77,0\%, respectivamente. Com relação à presença de coordenadores enfermeiros com especialização, esta foi menor em ambas as especialidades, com 58,0\% na obstetrícia e $64,4 \%$ na neonatologia, nos hospitais públicos, e $62,7 \%$ e $71,8 \%$ nos hospitais privados. Isso converge com a preocupação das mulheres com a falta de recursos humanos capacitados (33).

Embora pautado em métodos consistentes, o presente estudo apresenta algumas limitações, entre elas o tamanho da amostra e o viés de seleção, devido aos dados não terem sido coletados de forma ativa, tendo em vista que o perfil de mulheres que participam de uma consulta pública é potencialmente diferenciado por questões como acesso à rede de Internet e possibilidade de dedicar tempo, voluntariamente, para contribuir num texto dessa natureza. Logo, os resultados têm conclusões norteadoras para a implementação das diretrizes, mas não podemos afirmar que são representativos da opinião das mulheres brasileiras.

Ao interpretar essas contribuições, é importante considerar a possibilidade de serem provenientes, em sua maioria, de mulheres ativas na causa da assistência ao parto no Brasil. Por outro lado, pode-se igualmente especular que sejam contribuições de pessoas com maior disponibilidade de acesso a informações e, portanto, de desenvolvimento de crítica ao cuidado que é oferecido tanto no setor público quanto no setor privado.

A partir da análise realizada, observou-se que as barreiras para a implementação das Diretrizes de Assistência ao Parto, na percepção das mulheres que participaram dessa Consulta Pública, estavam concentradas nas dificuldades relacionadas a formação e cultura profissional, cultura social e questões políticas e de gestão. Resguardadas as limitações, considera-se que este estudo fornece importante contribuição para a implementação de políticas públicas para a saúde da mulher ao indicar potenciais pontos críticos para realização de futuros estudos ou intervenções, no sentido de transpor ou enfraquecer algumas barreiras para a implementação das Diretrizes de Assistência ao Parto Normal.

Contribuição dos autores. Todos os autores conceberam a ideia original e planejaram os estudos. ATV coletou os dados. ATV e DR analisaram os dados. ATV, JOB e DR realizaram a interpretação dos resultados. ATV redigiu a primeira versão do manuscrito. Todos os autores revisaram e aprovaram a versão final.

\section{Conflitos de interesse. Nada declarado pelos autores.}

Financiamento. Este estudo foi financiado pela Organização Pan-Americana da Saúde (OPAS/OMS), pelo Programa Especial de Pesquisa e Treinamento em Doenças Tropicais (TDR) e pela Aliança para Pesquisa em Políticas e Sistemas de Saúde (AHPSR) através de uma subvenção ao Projeto "Barreiras e estratégias para a implementação das Diretrizes do Parto Normal no Brasil [Chamada para propostas "Incorporação de pesquisas para os Objetivos de Desenvolvimento Sustentável (ER-SDG)]". Os financiadores não tiveram nenhum papel no desenho, análise, interpretação dos dados e redação do manuscrito.

Declaração. As opiniões expressas no manuscrito são de responsabilidade exclusiva dos autores e não refletem necessariamente a opinião ou política da RPSP/PAJPH ou da Organização Pan-Americana da Saúde (OPAS).

\section{REFERÊNCIAS}

1. Brasil, Ministério da Saúde. Sistema Único de Saúde. Disponível em: https://www.saude.gov.br/sistema-unico-de-saude Acessado em 11 de setembro de 2020.

2. Brasil, Agência Nacional de Saúde Suplementar (ANS). Beneficiários de planos privados de saúde, por cobertura assistencial
(Brasil - 2010-2020). Disponível em: http:/ / www.ans.gov.br/perfildo-setor/dados-gerais Acessado em 11 de setembro de 2020.

3. Brasil, Ministério da Saúde. Painel de Monitoramento de Nascidos Vivos segundo Classificação de Risco Epidemiológico (Grupos de Robson). Disponível em: http:/ /svs.aids.gov.br/dantps / 
centrais-de-conteudos/paineis-de-monitoramento/natalidade / grupos-de-robson/2018 Acessado em 14 de junho de 2020.

4. Oliveira RR de, Melo EC, Novaes ES, Ferracioli PLRV, Mathias TA de F. Factors associated to Caesarean delivery in public and private health care systems. Rev Esc Enferm USP. 2016;50(5):733-40. https://doi.org/10.1590/s0080-623420160000600004

5. Brasil, Ministério da Saúde. Gabinete do Ministro. Portaria 1459/2011. Disponível em: http://bvsms.saude.gov.br/bvs/saudelegis/gm/ 2011/prt1459_24_06_2011.html Acessado em novembro de 2020.

6. Brasil, Agência Nacional de Saúde Suplementar (ANS). Resolução Normativa 368/2015. Disponível em: https://www.ans.gov.br/ component/legislacao/?view=legislacao\&task=TextoLei\&format $=$ raw \&id=Mjg5 Mg==\#: :text=Disp $\% C 3 \%$ B5e $\% 20$ sobre $\% 20 \mathrm{o} \% 20$ direito $\% 20$ de,no $\% 20 \%$ C3\%A2mbito $\% 20$ da $\% 20$ sa $\%$ C3 $\%$ BAde $\% 20$ suplementar Acessado em novembro de 2020.

7. Brasil, Ministério da Saúde. Secretaria de Atenção à Saúde. Portaria $\mathrm{n}^{\circ}$ 353, de 14 de fevereiro de 2017. Aprova as Diretrizes Nacionais de Assistência ao Parto Normal. Disponível em: http:/ / conitec.gov. br/images/Protocolos/Diretrizes/DDT_Assistencia_PartoNormal. pdf Acessado em novembro de 2020.

8. Fervers B, Burgers JS, Voellinger R, Brouwers M, Browman GP, Graham ID, et al. Guideline adaptation: an approach to enhance efficiency in guideline development and improve utilisation. BMJ Qual Saf. 2011;20(3):228-36. http://dx.doi.org/10.1136/bmjqs.2010 .043257

9. Brasil. Lei 12 401/2011. Disponível em: http://www.planalto.gov. br/ccivil_03/_ato2011-2014/2011/lei/112401.htm\#: :text=Altera $\% 20$ a\%20Lei\%20n\%C2\%BA\%208.080,Sistema\%20\%C3\%9Anico $\% 20$ de $\% 20$ Sa $\%$ C3\%BAde $\% 20 \% 2$ D $\% 20$ SUS.\&text=\%E2\%80\%9CArt.\&text $=\mathrm{A} \% 20$ assist $\%$ C3 $\%$ AAncia $\% 20$ terap $\%$ C3 $\%$ AAutica $\% 20$ integral $\% 20$ a,do\%20inciso $\% 20 \mathrm{I} \% 20 \mathrm{do} \% 20$ art Acessado em novembro de 2020.

10. Brouwers MC, Kho ME, Browman GP, Burgers JS, Cluzeau F, Feder $\mathrm{G}$, et al. AGREE II: advancing guideline development, reporting and evaluation in health care. Can Med Assoc J. 2010;182(18): E839-42. https:/ / doi.org/10.1503/cmaj.090449

11. Harrison MB, Graham ID, van den Hoek J, Dogherty EJ, Carley ME, Angus V. Guideline adaptation and implementation planning: a prospective observational study. Implement Sci. 2013;8(1):49. https://doi.org/10.1186/1748-5908-8-49

12. Gagliardi AR, Marshall C, Huckson S, James R, Moore V. Developing a checklist for guideline implementation planning: review and synthesis of guideline development and implementation advice. Implement Sci. 2015;10(1):19. https://doi.org/10.1186/s13012-015 $-0205-5$

13. National Institute for Health and Care Excellence (NICE). Improving how patients and the public can help develop NICE guidance and standards. NICE; 2016.

14. Chapman E, Haby MM, Toma TS, de Bortoli MC, Illanes E, Oliveros MJ, et al. Knowledge translation strategies for dissemination with a focus on healthcare recipients: an overview of systematic reviews. Implement Sci. 2020;15(1):14. https://doi.org/10.1186/ s13012-020-0974-3

15. Brasil. Ministério da Saúde. Entendendo a Incorporação de Tecnologias em Saúde no SUS : como se envolver. Brasília: Ministério da Saúde; 2016.

16. Oliveira CF, Ribeiro AAV, Luquine Jr CD, de Bortoli MC, Toma TS, Chapman EMG, et al. Barreiras à implementação de recomendações para assistência ao parto normal: revisão rápida de evidências. Rev Panam Salud Publica. 2020;44:e132. https:/ / doi.org/10.26633/ RPSP.2020.132

17. Barreto JOM, Bortoli MC, Luquine Jr. CD, Oliveira CF, Toma TS, Ribeiro AAV, et al. Barreiras e estratégias para implementação de Diretrizes Nacionais do Parto Normal no Brasil. Rev Panam Salud Publica. 2020;44:e120. https://doi.org/10.26633/RPSP.2020.120

18. Comissão Nacional de Incorporação de Tecnologias no SUS (CONITEC). Contribuições da Consulta Pública - PCDT - Diretriz Nacional de Assistência ao Parto Normal-CONITEC. Consulta Pública no 1, de 8 de janeiro de 2016. Disponível em: http:/ / conitec.gov.br/images / Consultas/Contribuicoes/2016/CP_CONITEC_01_2016_PCDT_ Diretriz_Nacional_de_Assist\%C3\%AAncia_ao_Parto_Normal.pdf Acessado em novembro de 2020.
19. Bardin L. Análise de conteúdo. São Paulo: Edições 70; 2016.

20. Forero R, Nahidi S, De Costa J, Mohsin M, Fitzgerald G, Gibson $\mathrm{N}$, et al. Application of four-dimension criteria to assess rigour of qualitative research in emergency medicine. BMC Health Serv Res. 2018;18(120). https://doi.org/10.1186/s12913-018-2915-2

21. Brasil, Presidência da República. Lei 11 108/2005. Disponível em: http://www.planalto.gov.br/ccivil_03/_ato2004-2006/2005/lei/ 111108.htm Acessado em novembro de 2020.

22. Leal M do C, Pereira APE, Domingues RMSM, Filha MMT, Dias MAB, Nakamura-Pereira M, et al. Intervenções obstétricas durante o trabalho de parto e parto em mulheres brasileiras de risco habitual. Cad Saude Publica. 2014;30(suppl 1):S17-32. http://dx.doi. org/10.1590/0102-311X00151513

23. Leal M do C, Bittencourt S de A, Esteves-Pereira AP, Ayres BV da S, Silva LBRA de A, Thomaz EBAF, et al. Avanços na assistência ao parto no Brasil: resultados preliminares de dois estudos avaliativos. Cad Saude Publica. 2019;35(7). http:/ / dx.doi.org/10.1590 /0102-311x00223018

24. World Health Organization (WHO). The prevention and elimination of disrespect and abuse during facility-based childbirth. Genebra: WHO; 2015. Disponível em: https://apps.who.int/iris/bitstream/ handle/10665/134588/WHO_RHR_14.23_eng.pdf;jsessionid= CDC29B6324A27B5F7889C28A4C0A5990?sequence=1 Acessado em novembro de 2020.

25. World Health Organization (WHO). WHO recommendations: intrapartum care for a positive childbirth experience. Genebra: WHO 2018. Disponível em: https://www.who.int/publications/i/item /9789241550215 Acessado em novembro de 2020.

26. Lalonde A, Herschderfer K, Pascali-Bonaro D, Hanson C, Fuchtner C, Visser GHA. The International Childbirth Initiative: 12 steps to safe and respectful MotherBaby-Family maternity care. Int J Gynaecol Obstet. 2019 Jul;146(1):65-73. https://doi.org/10.1002/ ijgo.12844

27. Tobasía-Hege C, Pinart M, Madeira S, Guedes A, Reveiz L, Valdez-Santiago R, et al. Irrespeto y maltrato durante el parto y el aborto en América Latina: revisión sistemática y metaanálisis. Rev Panam Salud Publica. 2019;43:1. https://doi.org/10.26633/RPSP .2019 .36

28. Conselho Federal de Medicina (CFM). Resolução CFMno 2144/2016. Disponível em: https://portal.cfm.org.br/images/stories/pdf/ res21442016.pdf Acessado em novembro de 2020.

29. Cesar JA, Sauer JP, Carlotto K, Montagner ME, Mendoza-Sassi RA. Cesarean section on demand: a population-based study in Southern Brazil. Rev Bras Saude Materno Infant. 2017;17(1):99-105. https:/ / doi.org/10.11606/S1518-8787.2019053001466

30. World Health Organization (WHO). WHO recommendations: non-clinical interventions to reduce unnecessary caesarean sections. Genebra: WHO; 2018. Disponível em: https:/ /www.who.int/reproductivehealth/publications/non-clinical-interventions-to-reduce -cs/en/ Acessado em novembro de 2020.

31. Teixeira S, S Machado H. Who Caesarean Section Rate: Relevance and ubiquity at the present day - a review article. J Pregnancy Child Health. 2016;03(02). https://doi.org/10.4172/2376-127X.1000233

32. Diniz CSG, D'Orsi E, Domingues RMSM, Torres JA, Dias MAB, Schneck CA, et al. Implementação da presença de acompanhantes durante a internação para o parto: dados da pesquisa nacional Nascer no Brasil. Cad Saude Publica. 2014;30(suppl 1):S140-53. http:// dx.doi.org/10.1590/0102-311X00127013

33. Bittencourt SD de A, Reis LG da C, Ramos MM, Rattner D, Rodrigues PL, Neves DCO, et al. Estrutura das maternidades: aspectos relevantes para a qualidade da atenção ao parto e nascimento. Cad Saude Publica. 2014;(suppl 1):S208-19. https://doi.org/10.1590 /0102-311X00176913

Manuscrito recebido em 25 de julho de 2020. Aceito em versão revisada em 24 de setembro de 2020 . 


\section{Barriers to implementing childbirth recommendations in Brazil: the women's perspective}

ABSTRACT Objective. To identify barriers to the implementation of National Childbirth Guidelines in Brazil from the women's perspective.

Method. A descriptive exploratory study was performed using a qualitative approach and an interpretive perspective. The hermeneutic unit of analysis was established based on the contribution of users to a public online consultation about the National Childbirth Guidelines in Brazil, performed in 2016 by the National Committee for Health Technology Incorporation into the Unified Health System (CONITEC). Content analysis techniques were used to examine the answers provided to the following specific question: "Considering your local reality, what would hinder the implementation of this protocol or guideline?"

Results. Of 396 contributions recorded by CONITEC, 55 were included in the content analysis. The mean age of women was 31 years, with most self-declared as white (69\%) and living in the Southeast of Brazil (56.3\%). Coding revealed seven barrier categories, which were grouped into three families — barriers related to 1) professional training and culture (which highlighted the centrality of physicians, not women, in childbirth), 2) social culture (general population not well informed), and 3) political and management issues (little interest on the part of managers, lower physician compensation for vaginal childbirth vs. Caesarian section, and poor hospital infrastructure).

Conclusions. Aspects of professional training and culture, social culture, and political as well as management issues are critical points to be considered in future interventions aiming at overcoming or weakening the barriers to implementing childbirth recommendations in Brazil.

Keywords

Parturition; clinical protocols; implementation science; Brazil.

\section{Obstáculos para la aplicación de las recomendaciones de asistencia al parto normal en Brasil: perspectiva de las mujeres}

RESUMEN Objetivo. Determinar los obstáculos existentes para la aplicación de las directrices de asistencia al parto normal en Brasil desde la perspectiva de las mujeres.

Métodos. Se realizó un estudio descriptivo exploratorio, con un enfoque cualitativo y una perspectiva de investigación interpretativa. La unidad hermenéutica se construyó a partir de los aportes hechos por usuarias a una consulta pública en línea sobre las directrices nacionales de asistencia al parto normal realizada en el 2016 por la Comisión Nacional de Incorporación de Tecnologías (CONITEC) en el Sistema Único de Salud. Se utilizó la metodología de análisis del contenido para examinar específicamente las respuestas a la siguiente pregunta: considerando su realidad local, ¿qué dificultaría la implantación de este protocolo o de esta directriz?

Resultados. En el análisis del contenido se incluyeron 55 de los 396 aportes recibidos por la CONITEC. Las mujeres tenían una media de edad de 31 años y, en su mayoría, eran blancas (69\%) y residentes en la región Sudeste de Brasil (56,3\%). La codificación reveló siete categorías de obstáculos, agrupados en tres clases, a saber, obstáculos relacionados con 1) la formación y la cultura profesional (con hincapié en la centralidad de los médicos y no de las mujeres en el parto), 2) la cultura social (la falta de información por parte de la población) y 3) las cuestiones de política y gestión (la falta de interés de los gestores, la menor remuneración de los médicos que atienden el parto normal en comparación con quienes practican cesáreas y la falta de infraestructura hospitalaria).

Conclusiones. Los resultados mostraron que los aspectos relacionados con la formación y la cultura profesional, la cultura social y las cuestiones de política y gestión son puntos críticos que deben considerarse en la realización de intervenciones futuras con objeto de superar o reducir los obstáculos existentes para la aplicación de las recomendaciones de asistencia al parto normal en Brasil.

Palabras clave Parto; protocolos clínicos; ciencia de la implementación; Brasil. 